\title{
Tight Sylvian Cisterns Associated With Hyperdense Areas Mimicking Subarachnoid Hemorrhage on Computed Tomography
}

-Four Case Reports-

\author{
Satoru SHIMIZU*,***, Masataka ENDO*, Shinichi KAN**, Takao KITAHARA*, \\ Takashi OHWADA*, and Kiyotaka FUJII***
}

Departments of ${ }^{*}$ Critical Care and Emergency Medicine, ${ }^{* *}$ Radiology, and ${ }^{* * *}$ Neurosurgery, Kitasato University School of Medicine, Sagamihara, Kanagawa

\begin{abstract}
Four patients with supratentorial mass lesions (two chronic subdural hematomas, one acute epidural hematoma, and one acute subdural hematoma) showed hyperdense sylvian cisterns on computed tomography (CT). Association of subarachnoid hemorrhage was suspected initially, but was excluded by intraoperative observation or postoperative lumbar puncture. CT showed disappearance of the hyperdense areas just after evacuation of the mass lesions. The hyperdense areas are probably a result of the partial volume phenomenon or concentrations of calcium deposits rather than abnormally high hematocrit levels, which were not found in these patients.
\end{abstract}

Key words: computed tomography, intracranial mass, subarachnoid hemorrhage, cistern

\section{Introduction}

Computed tomography (CT) is an indispensable tool for neurological diagnosis, but occasionally leads to an incorrect diagnosis. Hyperdense areas in the cisterns are an important positive finding of subarachnoid hemorrhage ( $\mathrm{SAH}$ ). We describe four cases of peculiar hyperdense areas in the sylvian cistern in patients with supratentorial mass lesions, which were not associated with SAH.

\section{Case Reports}

Case 1: A 25-year-old male received a blow to his left temporal region with a stick and became comatose 2 hours after the trauma. On arrival at the hospital, he was unresponsive with a Glasgow Coma Scale score of 7 and dilated left pupil. Skull radiography showed a left temporal linear fracture. CT showed a thick acute epidural hematoma in the left temporal region and hyperdense areas in the bilateral sylvian cisterns (Fig. 1A, B). The hematoma was evacuated by emergency craniotomy. SAH was not observed in the sylvian fissure. CT obtained just after the surgery showed loosened cistern and disappearance of the sylvian hyperdense areas (Fig. 1C, D). He was discharged without neurological deficits.

Case 2: A 68-year-old male was hit by a bus when he was riding a bicycle. On arrival, he was comatose with a Glasgow Coma Scale score of 4 and dilated right pupil. CT showed a right acute subdural hematoma and a hyperdense area in the right sylvian cistern (Fig. 2A, B). Emergent craniectomy and removal of the hematoma was performed. SAH was not observed in the right sylvian cistern. CT obtained just after the surgery showed disappearance of the hyperdense area in the right sylvian cistern (Fig. 2C). His neurological status was not improved and he was transferred to another institute in the vegetative state.

Case 3: A 70-year-old male, who had no history of trauma, complained of severe headache and nausea continuing for 4 days. On admission to another hospital, he was conscious but showed slight weak-

Received October 17, 2000; Accepted August 11, 2001

Author's present address: S. Shimizu, M.D., Department of Neurosurgery, International Goodwill Hospital, Yokohama, Kanagawa, Japan. 

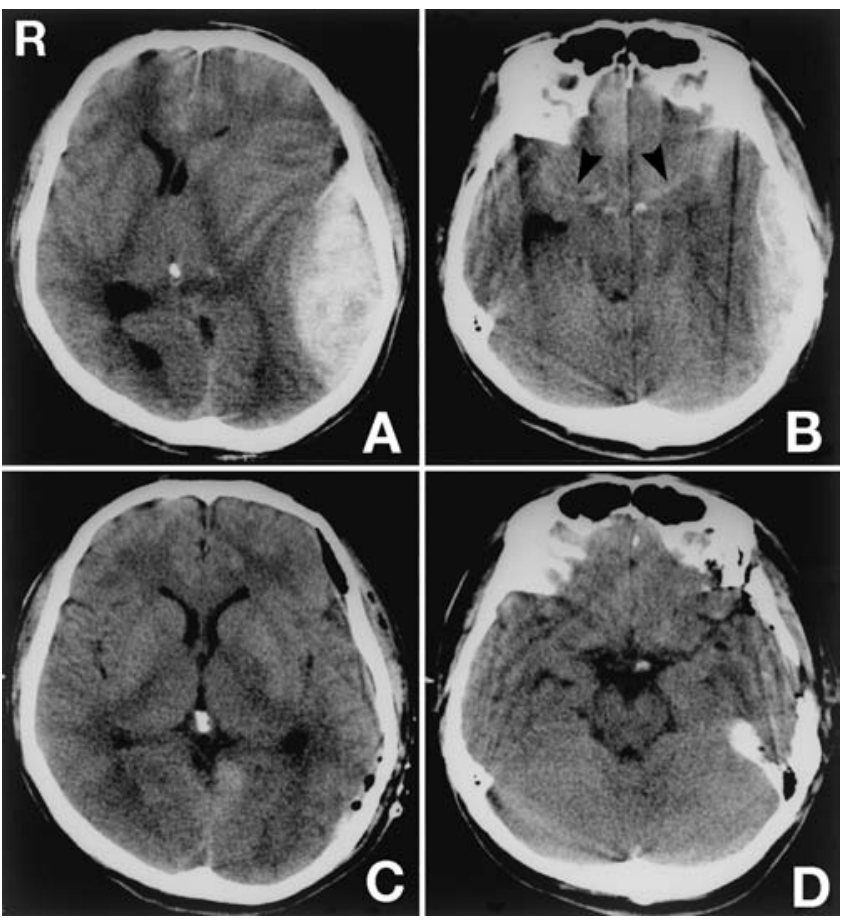

Fig. 1 Case 1. A, B: Initial computed tomography (CT) scans showing a left temporal acute epidural hematoma and hyperdense areas in the bilateral sylvian cisterns (arrowheads). C, D: CT scans just after the surgery showing the loosened cistern and disappearance of the sylvian hyperdense areas associated with removal of the hematoma. ness on the left. CT showed bilateral chronic subdural hematomas and hyperdense areas in the bilateral sylvian cisterns (Fig. 3A, B). CT with contrast medium showed these hyperdense areas were obscured by the opacified middle cerebral arteries (MCAs) (Fig. 3C). He was referred to our center. $\mathrm{SAH}$ was suggested and cerebral angiography was conducted to detect a bleeding source. No aneurysm or other vascular lesions were found. At surgery, significant old subdural hematomas were drained through burr holes on both sides. The brain was covered with the inner membrane of the hematoma. CT obtained just after the surgery showed disappearance of the hyperdense areas in the sylvian cisterns. No evidence of SAH was demonstrated by lumbar puncture 3 hours after the surgery. He made a complete recovery and was asymptomatic at discharge.

Case 4: A 43-year-old alcoholic male had complained of frontal headache for 3 days. He hit his head when he fell down 2 months before the complaint. On arrival, he complained of frontal headache and nausea. Neurological examination revealed no abnormality. CT showed bilateral chronic subdural hematomas and hyperdense areas in the bilateral sylvian cisterns (Fig. 4A). Magnetic resonance imaging showed vertically tightened sylvian fissure and basal cistern (Fig. 4B). Cerebral aneurysm and other vascular lesions were not found on cerebral angiography. Surgery revealed significant subdural, dark red blood through burr holes on both sides. The surface of the brain was intact. CT obtained just after the surgery showed disap-
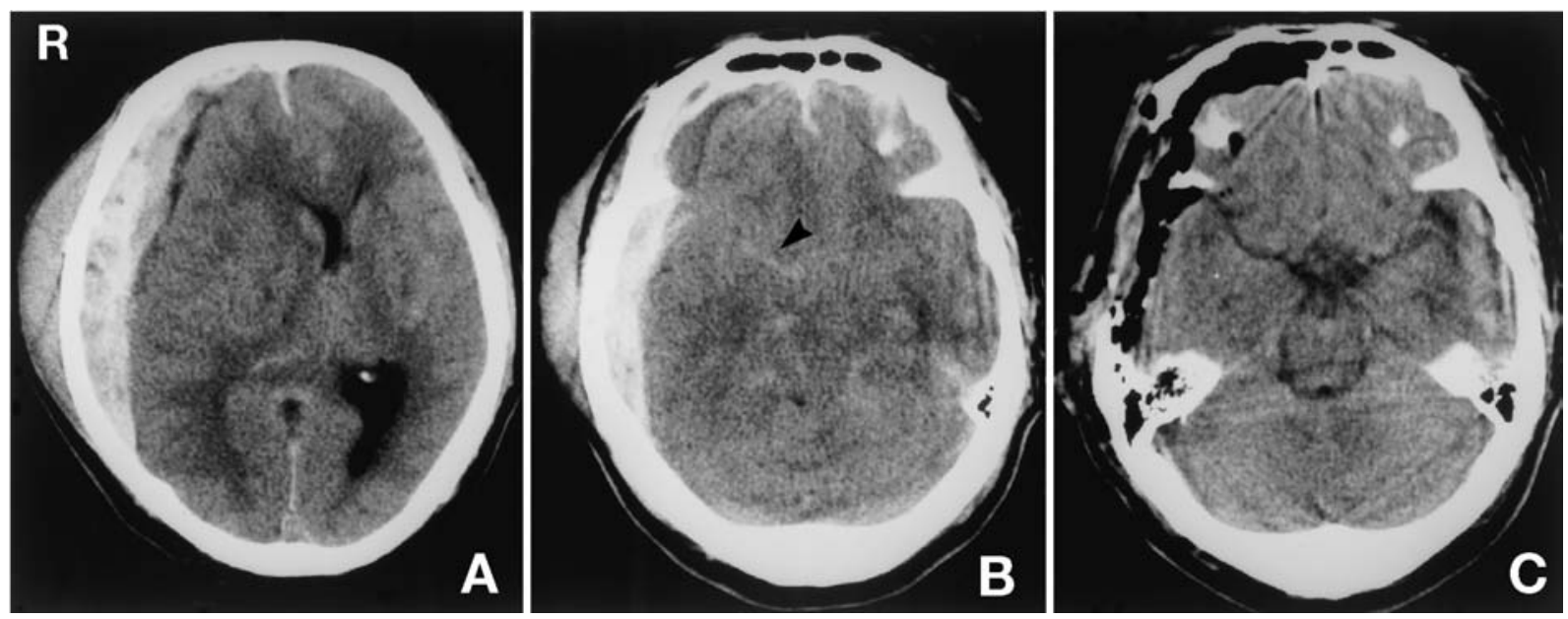

Fig. 2 Case 2. A, B: Initial computed tomography (CT) scans showing a right thick acute subdural hematoma and a hyperdense area in the right sylvian cistern (arrowhead). C: CT scan just after the surgery showing the loosened cistern and disappearance of the sylvian hyperdense area. 

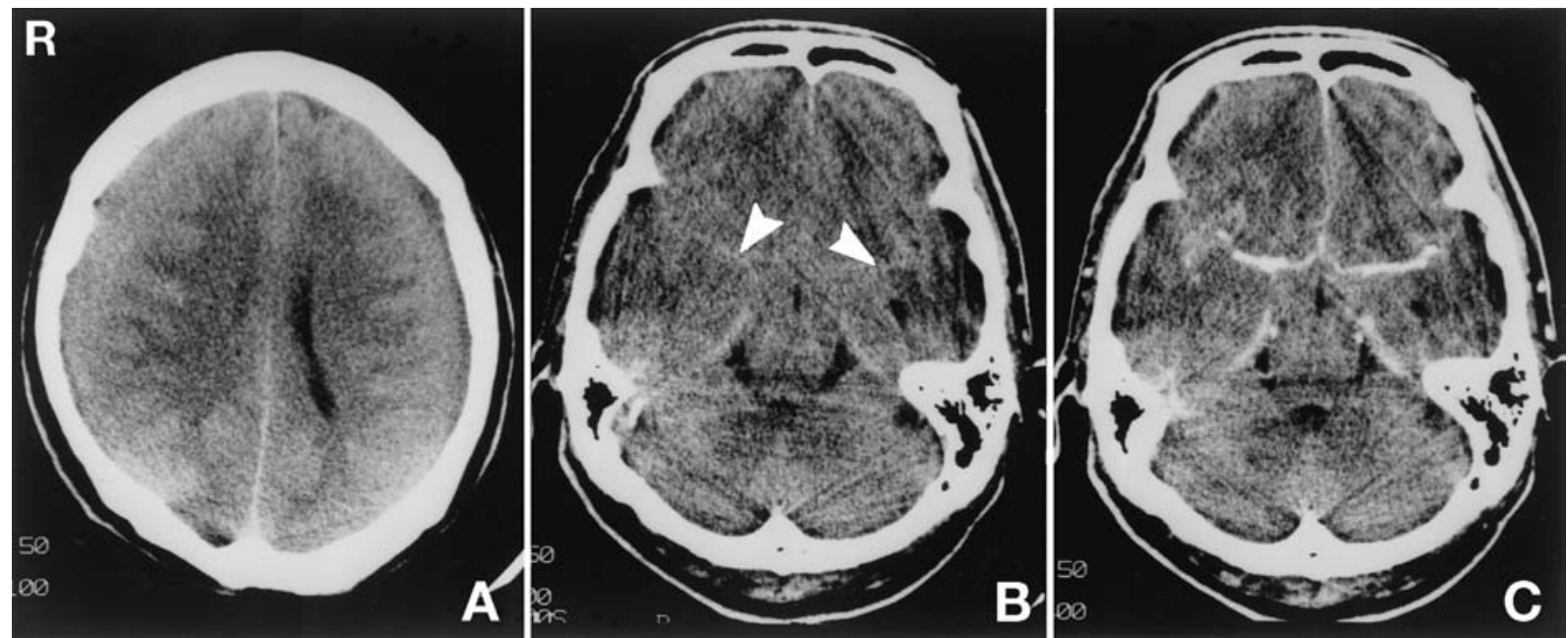

Fig. 3 Case 3. A, B: Initial computed tomography (CT) scans showing bilateral chronic subdural hematomas and hyperdense areas in the bilateral sylvian cisterns (arrowheads). C: CT scan showing that the hyperdense areas in the sylvian cisterns are obscured by the opacified middle cerebral arteries.

pearance of the increased density in the sylvian cistern (Fig. 4D). Cerebrospinal fluid obtained by lumbar puncture one day after the surgery revealed no evidence of SAH. He was discharged without neurological deficits.

\section{Discussion}

Four male patients with supratentorial mass lesions, aged from 25 to 70 years, had hyperdense areas in the sylvian cisterns on initial CT. Patient details are summarized in Table 1. CT used a ProSeed scanner (Yokogawa Medical Systems, Tokyo) with the following conditions: slices parallel to the orbitomeatal line, slice thickness of $5 \mathrm{~mm}$, window width of 80 Hounsfield units (HU), and window level of $40 \mathrm{HU}$. Technical information was unavailable for one referred case.

Hyperdense areas were observed in the basal part of the sylvian cistern, but not in the basal cistern. The hyperdense areas and mass lesions were located as unilateral mass and ipsilateral hyperdense area, unilateral mass and bilateral hyperdense areas, and bilateral masses and bilateral hyperdense areas. The extent of the hyperdense areas were proportional to the size of the hematoma in the patient with bilateral chronic subdural hematomas. Density measurements in the medial, middle, and lateral part of the hyperdense areas, available in three cases, were 42 to $52 \mathrm{HU}$ (mean $47.2 \mathrm{HU}$ ). Characteristics of the hyperdense areas are summarized in Table 2.

The density of the hyperdense areas (mean
Table 1 Summary of patients presenting with hyperdense sylvian cisterns on computed tomography, associated with supratentorial mass lesions

\begin{tabular}{|c|c|c|c|c|c|}
\hline $\begin{array}{l}\text { Case } \\
\text { No. }\end{array}$ & $\begin{array}{l}\text { Age/ } \\
\text { Sex }\end{array}$ & Diagnosis & $\begin{array}{l}\text { Surgery and } \\
\text { SAS findings }\end{array}$ & $\begin{array}{l}\text { Angiog- } \\
\text { raphy }\end{array}$ & $\begin{array}{l}\text { Lumbar } \\
\text { puncture }\end{array}$ \\
\hline 1 & $25 / \mathrm{M}$ & lt AEDH & $\begin{array}{l}\text { craniotomy, } \\
\text { no SAH }\end{array}$ & $\mathrm{NE}$ & $\mathrm{NE}$ \\
\hline 2 & $68 / \mathrm{M}$ & rt ASDH & $\begin{array}{l}\text { craniectomy, } \\
\text { no SAH }\end{array}$ & $\mathrm{NE}$ & $\mathrm{NE}$ \\
\hline 3 & $70 / \mathrm{M}$ & bil CSDH & $\begin{array}{l}\text { burr hole } \\
\quad \text { drainage, NE }\end{array}$ & normal & no SAH \\
\hline 4 & $43 / \mathrm{M}$ & bil CSDH & $\begin{array}{l}\text { burr hole } \\
\text { drainage, NE }\end{array}$ & normal & no SAH \\
\hline
\end{tabular}

AEDH: acute epidural hematoma, ASDH: acute subdural hematoma, CSDH: chronic subdural hematoma, NE: no examination, SAH: subarachnoid hemorrhage, SAS: subarachnoid space.

47.2 HU) in the sylvian cisterns were strongly indicative of hemorrhage based on accepted definitions of CT values.3) However, the appearance differed from typical SAH. The hyperdense areas in our cases had the following characteristics: coexistence of tight cisterns in the cranial base due to the supratentorial mass effect; location limited to the basal part of the sylvian cistern; obscured by the opacification of the MCA by contrast medium; and disappearance immediately after removal of the mass lesions.

The sylvian cisterns are tightened in patients with enlarging supratentorial masses. As a result of such 

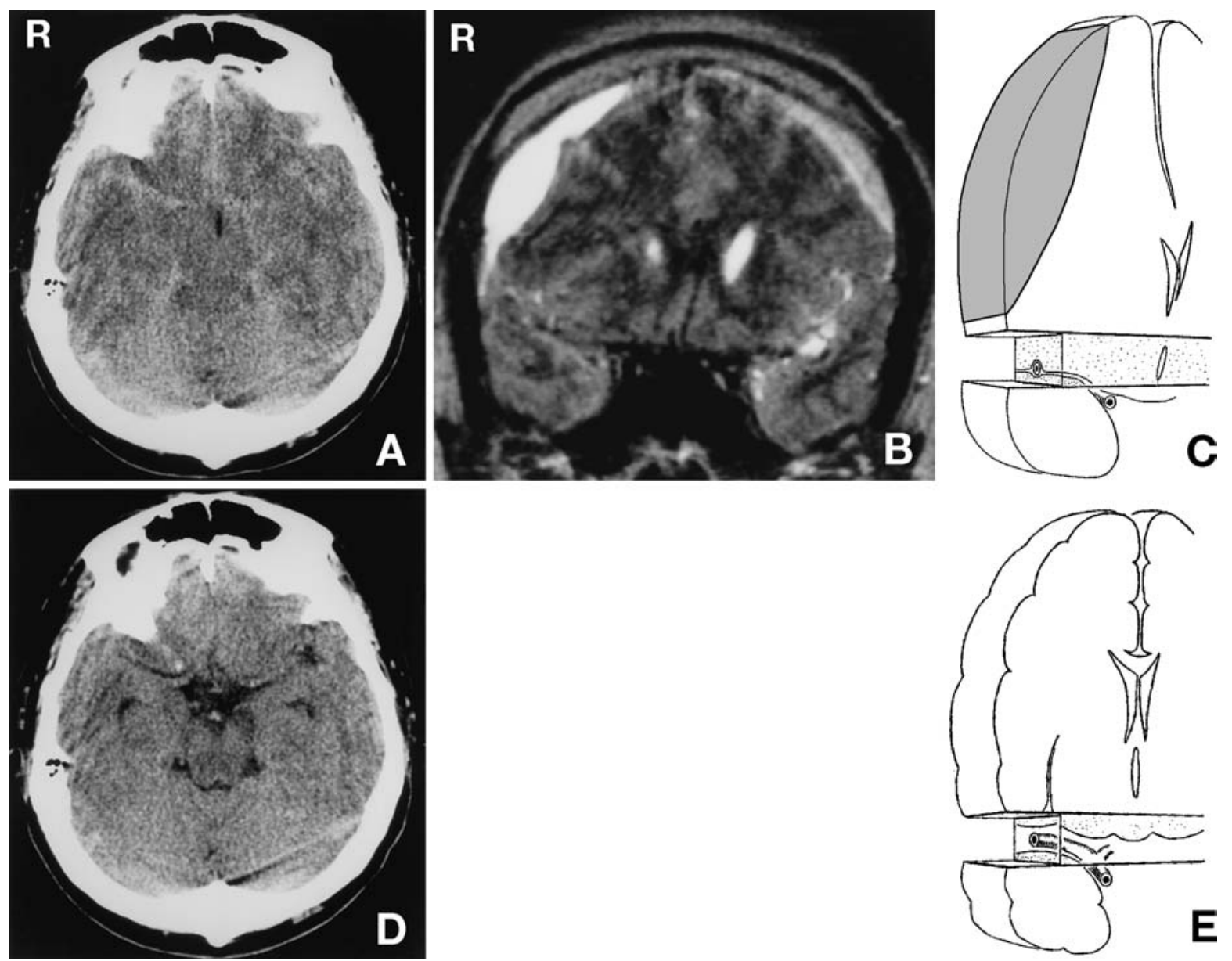

Fig. 4 Case 4. Relationships between configuration of the cisterns and corresponding computed tomography (CT) images. A-C: CT scan (A), coronal $\mathrm{T}_{2}$-weighted magnetic resonance image (B), and schematic diagram (C) prior to surgery. No cerebrospinal fluid space is shown in the right sylvian cistern due to compression by the large chronic subdural hematoma. The CT scan shows wider extent of hyperdense areas in the ipsilateral sylvian cistern than the contralateral side. The central portion of the slice is filled with the descended basal hypothalamus including the third ventricle. D, E: CT scan (D) and schematic diagram (E) after evacuation of the hematoma. The cistern is loosened and the hyperdense areas have disappeared.

Table 2 Characteristics of the hyperdense sylvian cisterns

\begin{tabular}{|c|c|c|c|c|c|c|c|}
\hline \multirow{3}{*}{ Case No. } & \multicolumn{6}{|c|}{ HDA in the sylvian cisterns ( $\mathrm{CT}$ value, $\mathrm{HU}$ ) } & \multirow{3}{*}{$\begin{array}{c}\text { Mean CT value } \\
\text { (HU) }\end{array}$} \\
\hline & \multicolumn{3}{|c|}{ Rt } & \multicolumn{3}{|c|}{ Lt } & \\
\hline & Lat & Mid & Med & Med & Mid & Lat & \\
\hline 1 & $+(47)$ & $+(50)$ & $+(47)$ & $+(47)$ & $+(50)$ & $+(47)$ & 48 \\
\hline 2 & $+(50)$ & $+(46)$ & $+(47)$ & - & - & - & 47.7 \\
\hline $3^{*}$ & + & + & + & + & + & + & \\
\hline 4 & $+(52)$ & $+(45)$ & $+(43)$ & $+(42)$ & - & - & 45.5 \\
\hline Total mean & & & & & & & 47.2 \\
\hline
\end{tabular}

${ }^{*} \mathrm{HU}$ measurement was unavailable. CT: computed tomography, HDA: hyperdense area, HU: Hounsfield unit, Lat: lateral, Mid: middle, Med: medial portion of the sylvian cistern, + : present, - : absent. 
morphological changes, the CT voxels mainly contain brain parenchyma and MCA, and less cerebrospinal fluid in the sylvian cistern portion (Fig. 4). This principle, called the "partial volume phenomenon," ${ }_{3}$ ) is a mechanism to express the hyperdense sylvian cistern. In addition, concentration of calcium deposits in the compressed MCA may also increase the apparent density of the area. The colocation of the hyperdense areas with the opacified MCA supports the idea that the compressed MCA is also contributory. Although a high level of hematocrit may indicate hyperdense MCA, ${ }^{1)}$ the present hyperdense areas were detected in the presence of normal or decreased hematocrit levels (26.6-45.4\%, mean $36.1 \%$ ).

The basal cisterns appeared as isodense structures, with caudally displaced basal hypothalami due to mass effects of supratentorial lesions. ${ }^{2,4)}$ The third ventricle may also be identified in this region. The hyperdense areas became hypodense, with the loose cisterns, immediately after evacuation of the mass lesions, due to reconstitution of the normal brain architecture. These hyperdense areas may be observed in patients with tight sylvian cisterns due to diseases other than hematoma, such as intracranial tumors, brain swelling, hydrocephalus, and adhesion between the lobes.

The current findings of hyperdense areas indicate prompt reduction of the intracranial mass, without additional examinations to confirm the presence and source of hemorrhage in the cistern. Probably, many clinicians have experienced the present types of images, but we would like to stress the importance of careful initial interpretation of CT scans for prompt and correct treatment.

\section{References}

1) Bakshi R, Mazziotta JC: Acute middle cerebral artery thrombosis demonstrated by cranial computed tomography. The "dense MCA” sign. Arch Neurol 55: 1577, 1998

2) Feldmann E, Gandy SE, Becker R, Zimmerman R, Thaler HT, Posner JB, Plum F: MRI demonstrates descending transtentorial herniation. Neurology 38: 697-701, 1988

3) Lange S, Grumme T, Meese W: Computerized Tomography of the Brain, ed 1. Berlin, Schering AG Berlin and Bergkamen, 1980, pp 2-7

4) Stovring J: Descending tentorial herniation: Findings on computed tomography. Neuroradiology 14: 101-105, 1977

Address reprint requests to: S. Shimizu, M.D., Department of Neurosurgery, International Goodwill Hospital, 1-28-1 Nishigaoka, Izumi-ku, Yokohama, Kanagawa 245-0006, Japan. 